\title{
The characteristics of plantar flexors and dorsiflexors strength in patients after complete three-bundle Achilles tendon reconstruction
}

A - preparing concepts

$\mathrm{B}$ - formulating methods

$\mathrm{C}$ - conducting research

$\mathrm{D}$ - processing results

E - interpretation and conclusions

$\mathrm{F}$ - editing the final version
Received: 2018-11-23 Accepted: 2019-04-04

\author{
Magdalena Syrek ${ }^{1 A, B, D-F}$, Robert Śmigielski ${ }^{2}, C, E, F$, Michał Staniszewski ${ }^{3} C, E, F$, \\ Michał Wychowański ${ }^{4}, E, F$
}

${ }^{1}$ Carolina Medical Center, Warsaw, Poland

${ }^{2}$ Mirai Institute of Rehabilitation, Warsaw, Poland

${ }^{3}$ Józef Piłsudski University of Physical Education in Warsaw, Department of Physical Education

4 Józef Piłsudski University of Physical Education in Warsaw, Department of Physiotherapy

https://doi.org/10.5114/areh.2019.85022

Key words: rehabilitation, general surgery, Achilles tendon, measurement 


\section{Introduction}

Achilles tendon rupture is an injury that often results from intensive physical activity performed without a proper warm-up and, above all, without stretching exercises. The occurrence of Achilles tendon ruptures is relatively high and is estimated at about 8 cases per 100,000 people. An average age of men who sustain this injury is 40 years of age $[1,2]$. Approximately $75-80 \%$ of all the Achilles tendon ruptures occur during physical activity such as football $(28 \%)$, tennis $(12 \%)$, volleyball (7\%), squash (7\%), jogging (7\%) and skiing (3\%). Twenty-five per cent of Achilles tendon injuries are not related to any sport $[3,4]$.

The calcaneal tendon forms the end of the triceps surae muscle which includes the gastrocnemius and the soleus. These muscle join to form the Achilles tendon, the tendon is therfore a three-bundle structure, which operates as one efficient mechanism under a variety of physiological conditions. The medial head of the gastrocnemius is attached above the medial femoral condyle, whilst the lateral head extends from the lateral epicondyle of the femur. The soleus is covered by the gastrocnemius and is attached to the posterior surface of the tibia and fibula. In contrast to the gastrocnemius, the soleus is a one-joint muscle. The soleus connects with other heads and together they form a strong calcaneal tendon on the calcaneal tuber. Particular muscle fibres within the Achilles tendon are arranged in a different way. The proximal part consists of fibres arranged in a parallel way. The first twists appear in the middle part whilst the fibres are totally twisted in the distal part about $5-6 \mathrm{~cm}$ above the upper edge of the heel bone; the twists range from 30 to 150 degrees(90 degrees on average) $[5,6,7]$.

Treatment approaches after an Achilles tendon rupture include a conservative treatment that involves, inter alia, immobilization for about 6-8 weeks or functional stiffness, which, in turn, excludes rehabilitation in the very first weeks. Such a treatment might increase the risk of re-rupture which occurs $10-30 \%$ significantly more often $(\mathrm{p}<0.05)$ than after surgical treatment [8]. The surgical methods of Achilles tendon reconstruction include Ma-Griffith percutaneous suture technique modified by Cretnik, Lindholm's technique using pedicled flap in the proximal stump, Lynn's plastic surgery with the use of the plantaris tendon and Kessler end-to-end suture technique modified by Krackow [9].
The Achilles rupture tendon surgery that is performed in the Carolina Medical Center in Warsaw involves reconstructing the three-bundle system and arranging the tendon fibres from the gastrocnemius and the soleus muscles in an appropriate manner $[6,7,10]$. Physiotherapy aimed to restore normal dynamic function of the Achilles tendon. Initially, analgesic and antiedematous treatment was applied and in the following stage the focus was on maintaining proper Achilles tendon glide. Attention was paid to proper stretching of muscles in the knee joint area, considering all three parts of the tendon and their attachments above and below the joint [12]. Further strengthening and proprioception training was provided in order to restore normal neuromuscular control and the ability to sense the joint position and kinaesthesia in the ankle [13]. The last stage of the post-operative treatment involved functional training that aimed to prepare the patient to return to physical activity in accordance with specific criteria [14].

The assessment of physical fitness of a person concerns patients and healthy people at every stage of their life, ranging from childhood [15] to the elderly age [16].

The main aim of this study was to assess the level of mobility of the operated limb one and two years after the Achilles tendon reconstruction surgery on the basis of the observed changes in plantar flexor and dorsiflexor muscle torque.

\section{Material and methods}

A group of 20 men were included in the research. The inclusion criteria were as follows: male gender, age $-35-65$ years, surgery after total Achilles tendon rupture or reoperation conducted within two weeks after the injury. Exclusion criteria: injuries of other joints in the lower limbs. All the participants received a referral from an attending orthopaedic surgeon to get examined. The characteristics of the study group are presented in Table 1. In all the cases, the Achilles tendon reconstruction was performed in the years 2009-2012. Nine patients had their right Achilles tendon ruptured whilst 11 of them injured their left tendon. In the case of 17 individuals, the tendon was ruptured during a sport activity, while three of them underwent reconstruction surgery after an unsuccessful attempt in another centre. 
Tab. 1. The characteristics of the study group

\begin{tabular}{|c|c|c|c|c|}
\hline & age & height $[\mathrm{cm}]$ & weight $[\mathrm{kg}]$ & BMI $\left[\mathrm{kg} / \mathrm{m}^{2}\right]$ \\
\hline mean & 47 & 182 & 88 & 26 \\
\hline SD & 8.3 & 7 & 12 & 3 \\
\hline $\min$ & 36 & 170 & 70 & 23 \\
\hline $\max$ & 65 & 196 & 110 & 32 \\
\hline
\end{tabular}

The rehabilitation process took place at the Carolina Medical Center in Warsaw and was conducted in accordance with a unified physiotherapeutic protocol which is applicable to all the patients in this clinic who have undergone Achilles tendon reconstruction, and has been created on the basis of the tendon healing principles [11] and suitably adjusted to the patients with different mobility levels and various anthropometric parameters. The rehabilitation was initiated on the second day after the surgery. At the beginning, the focus was on antiedematous treatment and on maintaining proper Achilles tendon glide. Ultrasound examination was performed 3,6 and 12 weeks after the surgery and proved the Achilles tendon reconstruction was progressing. A frontal cast protecting the dorsiflexors was used in the third week after the surgery and later on was replaced with a Walker Aircast shoe with $4-\mathrm{cm}$ heel pads. Simultaneously, weight-bearing exercises were initiated beginning from $25 \%$ of the body weight, gradually increasing by further $25 \%$ every week so that the patient experienced a full load on the operated limb within four weeks. In week 12, the patient underwent an ultrasound control to confirm whether the tendon redeveloped in a proper way in relation to the time that passed since the surgery was performed. At that stage, the patient also started to perform dynamic body stabilization exercises as well as eccentric-concentric exercises of lower limb muscles and prepared to do sports.

In the group of patients participating in the research, the rehabilitation lasted on average $6.3 \pm$ 1.9 months and took place three times a week for one hour. Functional tests were performed one and two years after the surgery. The patients completed a questionnaire in which they were asked about their dominant limb, which was defined as the limb that the patient uses for manipulative and precise operations such as passing a ball in the air. The other limb was treated as a non-dominant limb [19]. The scope of pain sensation in the operated tendon was also assessed by means of the VAS scale, in which 0 means complete absence of any pain, whilst 10 - the greatest imaginable pain [20]. The patients were also asked whether and to what extent they returned to physical activity from before the injury.

The measurement of the maximal torque developed in the ankle joint was performed by means of the Humac Norm device from an American company CSMi one and two years after the Achilles tendon reconstruction. The torques during plantar flexion and dorsiflexion in both lower limbs were measured both in a prone position with an extended knee (joint angle $\alpha=0$ ) and in a supine position with a flexed knee (joint angle $\alpha=50^{\circ}$ ) (Fig.1-2). The measurement positions were determined on the basis of the relevant literature $[17,18]$ and verified in our own pilot studies which showed that the torques in the Achilles tendon area significantly decreased in the position of knee joint flexed at $45-50^{\circ}$.

During the measurement in the static conditions, a study participant performed one trial test and then two muscle contractions with the maximum torque in the intermediate ankle joint position; the angle of the ankle joint was $\alpha=0$. Each muscle contraction lasted for approximately three seconds and was followed by a 10 -second break. The measurement under isokinetic conditions was performed for three angular velocities, i.e. $\omega=60 \%$ ( 5 repetitions $), \omega=$ $120^{\circ} / \mathrm{s}$ (8 repetitions) and $\omega=180^{\circ} / \mathrm{s}$ (10 repetitions) in a full painless range of motion. There was a 10-second break between the measurements. In order to avoid a significant influence of fatigue and to unify the measurement conditions of the maximal torque developed in the ankle joint during plantar flexion and dorsiflexion, it was assumed that the total time of maximal effort during each trial (cycle time: dorsiflexion - plantar flexion multiplied by the number of repetitions) should not exceed 10 seconds, which resulted from our own experience in measuring the maximal torque in static conditions. On this basis, an adequate number of repetitions was applied in each of the tests. The total duration of the

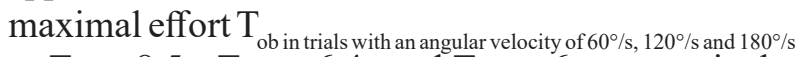
$\mathrm{T}_{\text {wob } 60}=8.5 \mathrm{~s}, \mathrm{~T}_{\mathrm{ob} 120}=6.4 \mathrm{~s}$ and $\mathrm{T}_{\mathrm{ob} 180}=6 \mathrm{~s}$, respectively. It seems that this type of approach made it possible to minimize the impact of endurance on the results of several repeated isometric efforts. The results of the measurements performed on the operated and non-operated limb were compared. 


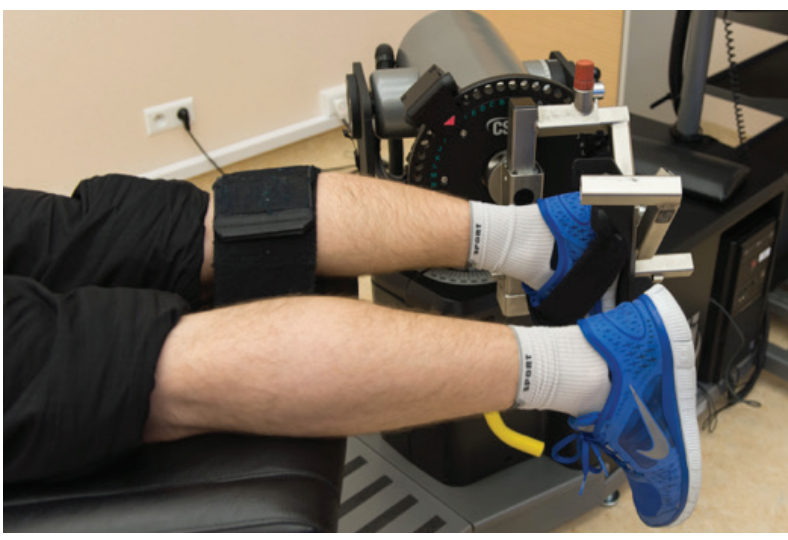

Fig.1. The position for measuring the torques during plantar flexion and dorsiflexion with an extended knee

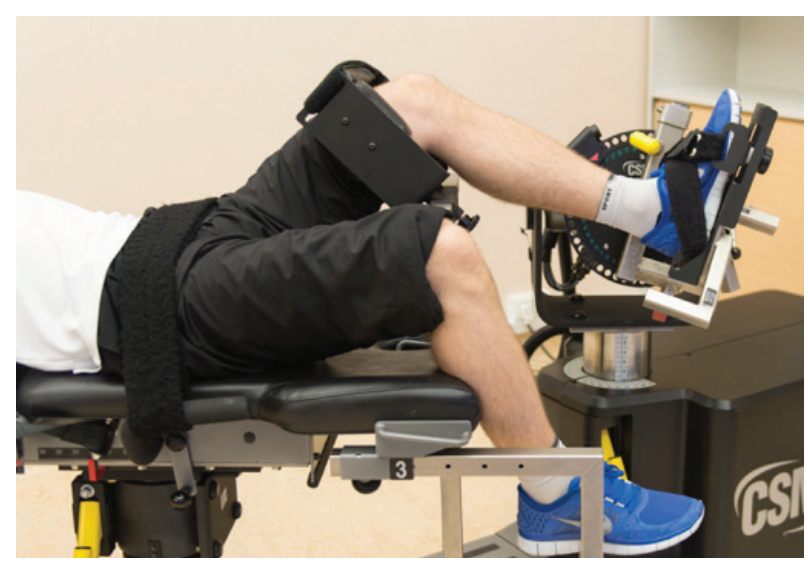

Fig.2. The position for measuring the torques during plantar flexion and dorsiflexion with the knee joint flexed to 50 degrees

The approval of the Bioethical Commission at the Medical Chamber in Warsaw as well as a written consent of the participants of the study were obtained prior to conducting the research.

Statistical analysis was performed with the use of StatSoft, Inc. (2011) STATISTICA version 10. The Shapiro-Wilk test was applied to check the normality of distribution of the data. Since some of the data did not show normal distribution, nonparametric Mann-Whitney U test was applied to compare the groups.

\section{Results}

The survey revealed that in the case of 18 patients (90\%), the right lower limb is the dominant one. The VAS showed that a mean pain score was at the level of $1.5 \pm 1.8$. Seventeen patients $(85 \%)$ returned to physical activity from before the surgery. Other people limited their activity to that of everyday living, explaining their choice by occasional pain in the Achilles tendon area and by the fear of yet another rupture.

The measurement of the plantar flexor and dorsiflexor muscle torques conducted in extendedknee position did not show any significant differences between the operated and non-operated limb one and two years after the surgery (Tab.2).

Tab. 2. Mean values of maximal torques (Mm) of plantar flexors in the operated and non-operated limb one and two years after the Achilles tendon reconstruction measured in the static conditions and for various angular velocities $\omega$ in the extended-knee position

\begin{tabular}{|c|c|c|c|c|c|}
\hline & \multicolumn{3}{|c|}{1 YEAR AFTER THE SURGERY } & \multirow{2}{*}{} \\
\hline \multirow{2}{*}{$\begin{array}{c}\boldsymbol{0} / \mathrm{s}] \\
\end{array}$} & $\begin{array}{c}\text { OPERATED LOWER } \\
\text { LIMB }\end{array}$ & $\begin{array}{c}\text { NON-OPERATED } \\
\text { LOWER LIMB }\end{array}$ & \\
\cline { 2 - 5 } & $\begin{array}{c}\mathrm{x} \pm \mathrm{SD} \\
{[\mathrm{Nm}]}\end{array}$ & $\begin{array}{c}\min -\mathrm{max} \\
{[\mathrm{Nm}]}\end{array}$ & $\overline{\mathrm{x}} \pm \mathrm{SD}[\mathrm{Nm}]$ & $\begin{array}{c}\text { min-max } \\
{[\mathrm{Nm}]}\end{array}$ & $\begin{array}{c}\text { DEFICIT } \\
{[\%]}\end{array}$ \\
\hline 0 & $90.6 \pm 23.4$ & $38.0-140.0$ & $97.9 \pm 22.5$ & $49.0-130.0$ & 7 \\
\hline 60 & $82.1 \pm 16.2$ & $45.0-117.0$ & $88 \pm 19.2$ & $52.0-122.0$ & 7 \\
\hline 120 & $53.2 \pm 10.8$ & $28.0-73.0$ & $58.4 \pm 13.3$ & $31.0-88.0$ & 9 \\
\hline 180 & $42.5 \pm 9.3$ & $23.0-61.0$ & $43.6 \pm 10.2$ & $23.0-69$ & 3 \\
\hline & 2 YEARS AFTER THE SURGERY & \\
\hline 0 & $90 \pm 21.2$ & $33.0-127.0$ & $102.3 \pm 21.9$ & $52.0-138.0$ & 12 \\
\hline 60 & $79.2 \pm 19.4$ & $35.0-123.0$ & $86.4 \pm 19.4$ & $41.0-123.0$ & 8 \\
\hline 120 & $52.8 \pm 13.2$ & $23.0-76.0$ & $54.7 \pm 13.3$ & $34.0-71.0$ & 3 \\
\hline 180 & $39.8 \pm 9.7$ & $20.0-58.0$ & $38.9 \pm 9.5$ & $19.0-56.0$ & 2 \\
\hline
\end{tabular}

The tests performed one year after the surgery in the flexed-knee position showed significantly lower values of the plantar flexor muscle torques in the operated limb measured under isokinetic conditions $(\mathrm{p}<0.05)$ compared to the results of the nonoperated limb. The results obtained two years after the reconstruction of the Achilles tendon showed that there were still some deficits of muscle torque developed during the tests conducted in static and isokinetic conditions. However, they were not statistically significant (Tab.3).

Comparing the measurements performed in the flexed-knee position one year after the surgery to the measurements carried out two years after the surgery, there was a significant decrease in the mean values of the plantar flexor muscle torques performed in the static and isokinetic conditions in both operated and non-operated limb (Tab.4).

The results of the measurements of dorsiflexion in the flexed-knee position are listed in Table 5. A decrease in the values of the dorsiflexor muscle torque was observed for both operated and nonoperated limb when measured in isokinetic conditions. 
Tab. 3. Mean values of maximal torques $(\mathrm{Mm})$ of plantar flexors in the operated and non-operated limb one and two years after the Achilles tendon reconstruction measured in the static conditions and for various angular velocities in the flexed-knee position

\begin{tabular}{|c|c|c|c|c|c|}
\hline \multirow{3}{*}{$\begin{array}{c}\boldsymbol{\omega} \\
{[\% / \mathrm{s}]}\end{array}$} & \multicolumn{4}{|c|}{1 YEAR AFTER THE SURGERY } & \\
\hline & \multicolumn{2}{|c|}{ OPERATED LOWER LIMB } & \multicolumn{2}{|c|}{ NON-OPERATED LOWER LIMB } & \\
\hline & $\overline{\mathrm{x}} \pm \mathrm{SD}[\mathrm{Nm}]$ & $\begin{array}{c}\min -\max \\
{[\mathrm{Nm}]} \\
\end{array}$ & $\overline{\mathrm{x}} \pm \mathrm{SD}[\mathrm{Nm}]$ & $\begin{array}{c}\min -\max \\
{[\mathrm{Nm}]}\end{array}$ & DEFICIT [\%] \\
\hline 0 & $88.4 \pm 25.0$ & $42.0-164.0$ & $97.8 \pm 31.1$ & $52.0-176.0$ & 10 \\
\hline 60 & $72.6 \pm 15.9^{*}$ & $31.0-107.0$ & $86.9 \pm 19.9^{*}$ & $45.0-137.0$ & 16 \\
\hline 120 & $53.3 \pm 11.8^{*}$ & $28.0-84.0$ & $62.2 \pm 16.6^{*}$ & $33.0-104.0$ & 14 \\
\hline \multirow[t]{2}{*}{180} & $43.3 \pm 9.3^{*}$ & $28.0-65.0$ & $49.8 \pm 11.5^{*}$ & $33.0-79$ & 13 \\
\hline & \multicolumn{4}{|c|}{2 YEARS AFTER THE SURGERY } & \\
\hline 0 & $76.7 \pm 16.5$ & $35.0-108.0$ & $80 \pm 22.4$ & $47.0-119.0$ & 4 \\
\hline 60 & $65.3 \pm 16.1$ & $24.0-81.0$ & $74.2 \pm 19.1$ & $30.0-106.0$ & 12 \\
\hline 120 & $46.2 \pm 10.5$ & $35.0-71.0$ & $52.6 \pm 12.2$ & $20.0-65.0$ & 12 \\
\hline 180 & $37.8 \pm 9.8$ & $15.0-56.0$ & $41.7 \pm 8$ & $18.0-52.0$ & 9 \\
\hline
\end{tabular}

* statistically significant differences $(\mathrm{p}<0.05)$

Tab. 4. Mean values of maximal plantar flexor muscle torque $(\mathrm{Mm})$ in the operated and non-operated limb in the static conditions and for various angular velocities $\omega$ in the flexed-knee position measured one and two years after the Achilles tendon reconstruction

\begin{tabular}{|c|c|c|c|c|c|}
\hline \multirow{3}{*}{$\begin{array}{c}\boldsymbol{\omega} \\
{[\% / s]}\end{array}$} & \multicolumn{4}{|c|}{ OPERATED LOWER LIMB } & \multirow[b]{3}{*}{ DEFICIT [\%] } \\
\hline & \multicolumn{2}{|c|}{1 YEAR AFTER THE SURGERY } & \multicolumn{2}{|c|}{2 YEARS AFTER THE SURGERY } & \\
\hline & $\overline{\mathrm{x}} \pm \mathrm{SD}[\mathrm{Nm}]$ & $\underset{[\mathrm{Nm}]}{\min -\max }$ & $\overline{\mathrm{x}} \pm \mathrm{SD}[\mathrm{Nm}]$ & $\underset{[\mathrm{Nm}]}{\min -\max }$ & \\
\hline 0 & $88.4 \pm 25^{*}$ & $42.0-164.0$ & $76.7 \pm 16.5^{*}$ & $35.0-108.0$ & 13 \\
\hline 60 & $72.6 \pm 15.9^{*}$ & $31.0-107.0$ & $65.3 \pm 16.1^{*}$ & $24.0-81.0$ & 10 \\
\hline 120 & $53.3 \pm 11.8^{*}$ & $28.0-84.0$ & $46.2 \pm 10.5^{*}$ & $35.0-71.0$ & 13 \\
\hline \multirow[t]{2}{*}{180} & $43.3 \pm 9.3^{*}$ & $28.0-65.0$ & $37.8 \pm 9.8^{*}$ & $15.0-56.0$ & 13 \\
\hline & \multicolumn{4}{|c|}{ NON-OPERATED LOWER LIMB } & \\
\hline 0 & $97.8 \pm 31.1^{*}$ & $52.0-176.0$ & $80 \pm 22.4^{*}$ & $47.0-119.0$ & 18 \\
\hline 60 & $86.9 \pm 19.9^{*}$ & $45.0-137.0$ & $74.2 \pm 19.1 *$ & $30.0-106.0$ & 15 \\
\hline 120 & $62.2 \pm 16.6^{*}$ & $33.0-104.0$ & $52.6 \pm 12.2 *$ & $20.0-65.0$ & 15 \\
\hline 180 & $49.8 \pm 11.5^{*}$ & $33.0-79$ & $41.7 \pm 8^{*}$ & $18.0-52.0$ & 16 \\
\hline
\end{tabular}

* statistically significant differences $(\mathrm{p}<0.05)$

Tab. 5. Mean values of maximum dorsiflexor muscle torque $(\mathrm{Mm})$ in the operated and non-operated limb in the static conditions and for various angular velocities $\omega$ in the flexed-knee position measured one and two years after the Achilles tendon reconstruction

\begin{tabular}{|c|c|c|c|c|c|}
\hline \multirow{3}{*}{$\begin{array}{c}\boldsymbol{\omega} \\
{[\% / \mathrm{s}]}\end{array}$} & \multicolumn{4}{|c|}{ OPERATED LOWER LIMB } & \multirow[b]{3}{*}{ DEFICIT [\%] } \\
\hline & \multicolumn{2}{|c|}{1 YEAR AFTER THE SURGERY } & \multicolumn{2}{|c|}{2 YEARS AFTER THE SURGERY } & \\
\hline & $\begin{array}{c}\overline{\mathrm{x}} \pm \mathrm{SD} \\
{[\mathrm{Nm}]}\end{array}$ & $\underset{[\mathrm{Nm}]}{\min -\max }$ & $\begin{array}{c}\overline{\mathrm{x}} \pm \mathrm{SD} \\
{[\mathrm{Nm}]}\end{array}$ & $\underset{[\mathrm{Nm}]}{\min -\max }$ & \\
\hline 0 & $35.3 \pm 9.5$ & $18.0-54.0$ & $37.2 \pm 9.1$ & $12.0-50.0$ & 5 \\
\hline 60 & $26.6 \pm 4.5^{*}$ & $15.0-39.0$ & $24.4 \pm 5.0 *$ & $12.0-34.0$ & 9 \\
\hline 120 & $18.6 \pm 3.5^{*}$ & $11.0-28.0$ & $16.1 \pm 3.3^{*}$ & $9.0-23.0$ & 13 \\
\hline \multirow[t]{2}{*}{180} & $16.8 \pm 2.4^{*}$ & $11.0-22.0$ & $14.4 \pm 3.0 *$ & $8.0-19.0$ & 14 \\
\hline & \multicolumn{4}{|c|}{ NON-OPERATED LOWER LIMB } & \\
\hline 0 & $39.9 \pm 7.8$ & $16.0-53.0$ & $38.5 \pm 7.5$ & $14.0-46.0$ & 4 \\
\hline 60 & $26.6 \pm 5.6^{*}$ & $11.0-33.0$ & $23.6 \pm 4.8^{*}$ & $9.0-27.0$ & 11 \\
\hline 120 & $17.1 \pm 3.2 *$ & $9.0-23.0$ & $15.7 \pm 3.2 *$ & $8.0-19.0$ & 8 \\
\hline 180 & $15.7 \pm 2.8^{*}$ & $9.0-19.0$ & $13.8 \pm 3.6^{*}$ & $5.0-18.0$ & 12 \\
\hline
\end{tabular}

* statistically significant differences $(\mathrm{p}<0.05)$ 


\section{Discussion}

The gastrocnemius, which constitutes part of the Achilles tendon, is a two-joint muscle that crosses both the knee joint and the ankle joint. Thus, the knee joint bending angle may affect the torque of the Achilles tendon during plantar flexion of the ankle joint in both static and isokinetic tests. The examination of healthy individuals resulted in obtaining lower values of muscle torque of the gastrocnemius in a static measurement in the flexed-knee position as compared to the extendedknee position [21-23]. According to the authors, these values were lower due to the shortened muscle and thus a lower possibility to generate muscle torque because a significant shortening of the gastrocnemius along with an increased knee joint angle were observed during the ultrasound examination. Similarly, it was noticed that the length of the soleus, which is attached below the knee, remained unchanged regardless of the angle changes and therefore, it was concluded that muscle torque did not depend on the knee joint angle [24].

Comparable values of the plantar flexor muscle torque both in the extended-knee position and flexed-knee position were observed during the measurement conducted under isokinetic conditions at a velocity of $180^{\circ} / \mathrm{s}$ [25-27] whilst the muscle torque in the flexed-knee position during plantar flexion at the velocity of $60 \% \mathrm{~s}$ exceeded the values obtained in the extended-knee position [28].

The results of EMG test are different though. Both in a static [29] and dynamic measurement [30] the gastrocnemius exhibited lower functional potential in the flexed-knee position because of the maximum shortening of its muscle fibres [31]. Moreover, Wahakara et al. measured the potential separately for the medial and lateral head of the gastrocnemius. They revealed significantly higher values for the medial head both in a static and dynamic measurement in the extended-knee position. A similar tendency was observed in the case of the lateral head; however, the EMG of the soleus and tibia did not change regardless of the knee bending angle. What is more, the torque difference between the extended and flexed knee was lower in the dynamic tests than in the static ones [27].

In this paper we analysed the measurement of the plantar flexor and dorsiflexor muscle torque in patients who sustained a complete Achilles tendon rupture and were treated with three-bundle anatomical tendon reconstruction, which involves suturing individual tendon fibers at several levels $[6,7]$. The main focus of the relevant subject literature is on the measurement of the tendon strength after end-to-end suture and percutaneous reconstruction [32]. In the case of end-to-end reconstruction, the scar occurring in the area of the Achilles tendon midsection leads to the limitation of motion in the area where the tendon is the thinnest and must quickly transfer the energy generated by the muscle during isokinetic testing. In the case of the anatomical reconstruction of the tendon, i.e. by suturing the fibers at different levels, one restriction area can be avoided and thus higher values can be generated during the muscle torque test.

The results of the study have shown a slight decrease in the plantar flexor muscle torque in the extended-knee position. In the dynamic measurement at an angular velocity of $\omega=180^{\circ} / \mathrm{s}$, the deficit fluctuated at around $2 \%$ as compared to the non-operated lower limb and was similar to the results obtained by Chan et al., who revealed $4 \%$ after end-to-end suture [33]. Both groups were similar in terms of age and duration of rehabilitation, but the measurement was carried out approximately 40 months after the surgery. On the other hand, Naim et al. showed a lower tendon deficit, which in the $120 \%$ measurement in the extended-knee position amounted to about $1.8 \%$ [32]. The authors proved that there were no significant differences in the plantar flexor muscle torque in the operated and non-operated limb when measured at $180^{\circ} \%$ s. Only in the measurement at $30 \%$ s there was a clear decrease in muscle torque which proved that the reconstructed tendon was not strong enough to overcome the huge lever resistance when moving at a constant speed of $30 \%$ s. This was also confirmed by Chan's research in which a strength deficit of $18 \%$ was obtained when measured at $60 \%$ s [33].

A significant weakness of plantar flexors in the operated limb as compared to its non-operated counterpart when measured in the flexed-knee position one year after the surgery was observed in this study. Moller et al. also measured muscle torque in the ankle in two measurement positions: knee extended and flexed at 90 degrees in a sitting position. They did not show any significant differences between the plantar flexor of the operated limb as compared to the non-operated one a year and two years after the surgery [35]. The patients were treated with the end-to-end suture technique and underwent 
a more demanding rehabilitation protocol which allowed a full load as early as 8 weeks after the surgery and an early implementation of concentric and eccentric exercises. The authors did not provide any evidence which inclined them to increase the scope of exercises during the rehabilitation process (for example ultrasound examination), which may be proved by one Achilles re-rupture in 18 weeks. Different results were presented by Jallageas et al. who demonstrated the weakening of the plantar flexors with a knee flexed at $18 \%$ in muscle torque tests at $30 \%$ s and $120 \%$ s. These tests were carried out 15 months after the surgery performed with the use of the Kessler's method [36] and confirmed the results of our study in which a similar tendency of torque deficit was revealed in all the three applied angular velocities.

One of the few authors who carried out the measurement of the plantar flexor muscle torque after the three-bundle Achilles reconstruction surgery, without reconstructing the anatomical system and fibre rotation, was Bevoni et al. [37]. The study was carried out in the extended-knee position at three angular velocities of $60 \% \mathrm{~s}, 120^{\circ} / \mathrm{s}$ and $180^{\circ} / \mathrm{s}$. The authors did not reveal any significant differences between the operated and non-operated limb, which confirmed the results of our study. The rehabilitation protocol differed from the one that was applied for the purpose of our study and allowed full load as early as 6 weeks after the surgery [37].

Despite the observed torque deficits $(p<0.05$ within the operated limb in relation to its nonoperated counterpart one and two years after the surgery, the patients did not feel any muscle weakness and the majority of them returned to practicing sports. A similar fact was confirmed by the authors of other publications who showed there were still some differences in muscle torque between the operated and non-operated limb 2 and even 10 years after the injury $[38,39,40]$.

\section{Conclusions}

According to the study, the angular position of the knee joint affects the plantar flexor muscle torque in the operated limb. When measured in the extended-knee position, similar parameters of muscle torque were observed in the operated limb in relation to the non-operated one both in the first and second examination. However, the difference in the muscle torque with the flexed knee is visible both one and two years after the surgery. A slight decrease in torque value as well as a difference between the non-operated and operated limb were observed in the measurement conducted two years after the surgery. Relating the results of muscle torque measurement to the results of the research on tissue healing and collagen reconstruction in the Achilles tendon, which lasts up to two years [11], it cannot be determined that the process of tissue healing affects changes in muscle torque. However, a hypothesis can be formulated that the reduction in the mean torque values in both operated and non-operated limbs stems from the limitation of the level of physical activity among the examined patients, which might have its roots in the need for postoperative treatment and lifestyle changes with limited physical activity. Therefore, additional training should be carried out after the rehabilitation has been completed and should last at least until that period. Particular attention should be paid to performing dynamic exercises in the flexed-knee position as they help to rebuild deep muscles such as the soleus. During the rehabilitation process, dorsiflexor training should not be neglected either as the deficit was visible therein both one and two years after the surgery.

A significant difference in the plantar flexor muscle torque is an important criterion for people who decide whether to return to practicing intense physical activity. On the basis of the conducted tests, it can be concluded that before making such a decision, it is necessary to perform isokinetic measurement in both extended- and flexed-knee positions. Isometric tests conducted after one and two years were similar to a healthy limb in an extended-knee position. Nevertheless, the deficit in the flexed-knee position was still observed. The above observations also allow to conclude that the measurement performed in static conditions cannot be an adequate method for the functional assessment of patients who have undergone the reconstruction of the Achilles tendon. 


\section{References}

1. Suchak AA, Bostick G, Reid D, Blitz S, Jomha N. The incidence of Achilles tendon ruptures in Edmonton, Canada. Foot Ankle Int. 2005 Nov;26(11):932-6.

2. Leppilahti J, Puranen J, Orava S, Incidence of Achilles tendon rupture. Acta Orthop Scand, 1996 Jun;67(3):277-9.

3. Strauss EJ, Ishak C, Jazrawi L, Sherman O, Rosen J. Operative treatment of acute Achilles tendon ruptures: An institutional review of clinical outcomes. Injury 2007 Jul;38(7):832-8.

4. Winter E, Weise K, Weller S, Ambacher T. Surgical repair of Achilles tendon rupture. Comparison of surgical with conservative treatment. Arch Orthop Trauma Surg. 1998;117(6-7):364-7.

5. Ciszek B, Śmigielski R, Wewnętrzna struktura ścięgna piętowego (Achillesa). Acta Clinica 2003;3(3):210-15.

6. Szaro P, Witkowski G, Smigielski R, Krajewski P, Ciszek B. Fascicles of the adult human Achilles tendon - an anatomical study. Ann Anat. 2009;191(6):586-93.

7. Śmigielski R. Management of partial tears of the gastro-soleus complex. Clin Sports Med. 2008;27:219-29.

8. Cetti R, Christensen SE, Ejsted R. Operative versus nonoperative treatment of Achilles tendon rupture. A prospective randomized study and review of the literature. Am J Sports Med. 1993 Nov-Dec;21(6):791-9.

9. Adamczyk G, Boszczyk A, Luboiński Ł. Metaanaliza metod leczenia operacyjnego całkowitych uszkodzeń ścięgna Achillesa, Acta Clinica 2003;3(4):331-38.

10. Pierre-Jerome C, Moncayo V, Terk MR. MRI of the Achilles tendon: A comprehensive review of the anatomy, biomechanics, and imaging of overuse tendinopathies. Acta Radiol. 2010 May;51(4):438-54.

11. Sharma P, Maffulli N. Tendon injury and tendinopathy: healing and repair. J Bone Joint Surg Am. 2005 Jan;87(1):187-202.

12. Strom AC, Casillas MM. Achilles Tendon Rehabilitation. Foot Ankle Clin. 2009 Dec;14(4):773-82.

13. Willems T, Witvrouw E, Verstuyft J, Vaes P, Clercq D. Proprioception and muscle strength in subjects with a history of ankle sprains and chronic instability. J Athl Train. 2002 Dec;37(4):487-93.

14. Saxena A, Ewen B, Maffulli N. Rehabilitation of the operated achilles tendon: parameters for predicting return to activity. J Foot Ankle Surg. 2011 Jan-Feb;50(1):37-40.

15. Świst A, Graff K, Chałubińska D, Zielińska A, Łukaszewska A. The effects of interventions implemented during a rehabilitation camp on changes in motor skills parameters and body mass in patients with simple obesity. Adv Rehab. 2017;(3):5-15.

16. Romero-Corral A, Somers VK, Sierra-Johnson J, Thomas RJ, Collazo-Clavell ML, Korinek J. et al. Accuracy of body mass index in diagnosing obesity in the adult general population. Int J Obes (Lond). 2008 Jun;32(6):959-66.

17. Fugl-Meyer AR, Sjöström M, Wählby L. Human plantar flexion strength and structure. Acta Physiol Scand. 1979 Sep;107(1): 47-56.

18. Orishimo KF, Burstein G, Mullaney MJ, Kremenic IJ, Nesse M, McHugh MP, Lee SJ. Effect of knee flexion angle on Achilles tendon force and ankle joint plantarflexion moment during passive dorsiflexion. J Foot Ankle Surg. 2008 Jan-Feb;47(1):34-9.

19. Peters M. Footedness: asymmetries in foot preference and skill and neuropsychological assessment of foot movement. Psychol Bull. 1988 Mar;103(2):179-92.

20. Jensen MP, Chen C, Brugger AM. Interpretation of visual analog scale ratings and change scores: a reanalysis of two clinical trials of postoperative pain. J Pain. 2003 Sep;4(7):407-14.

21. Maganaris CN. Force-length characteristics of the in vivo human gastrocnemius muscle. Clin Anat. 2003 May;16(3):215-23.

22. Herzog W, Read LJ, Ter Keurs HE. Experimental determination of force-length relations of intact human gastrocnemius muscles. Clin Biomech (Bristol, Avon). 1991 Nov;6(4):230-8.

23. Kawakami Y, Kumagai K, Huijing PA, Hijikata T, Fukunaga T. The length-force characteristics of human gastrocnemius and soleus muscles in vivo. In: Herzog W, editors. Skeletal Muscle Mechanics: Mechanisms to Function. Wiley, New York; 2000. p. 327-41.

24. Kawakami Y, Ichinose Y, Fukunaga T. Architectural and functional features of human triceps surae muscles during contraction. J Appl Physiol (1985). 1998 Aug;85(2):398-404.

25. Fugl-Meyer AR, Sjöström M, Wählby L. Human plantar flexion strength and structure. Acta Physiol Scand. 1979 Sep;107(1): 47-56.

26. Svantesson U, Ernstoff B, Bergh P, Grimby G. Use of a Kin-Com dynamometer to study the stretch-shortening cycle during plantar flexion. Eur J ApplPhysiolOccup Physiol. 1991;62(6):415-9.

27. Wakahara T, Kanehisa H, Kawakami Y, Fukunaga T. Fascicle behavior of medial gastrocnemius muscle in extended and flexed knee positions. J Biomech. 2007;40(10):2291-8.

28. Carpentier A, Duchateau J, Hainaut K. Velocity-dependent muscle strategy during plantarflexion in humans. J Electromyogr Kinesiol.1996 Dec;6(4):225-33.

29. Cresswell AG, Löscher WN, Thorstensson A. Influence of gastrocnemius muscle length on triceps surae torque development and electromyographic activity in man. Exp Brain Res.1995;105(2):283-90. 
30. Price TB, Kamen G, Damon BM, Knight CA, Applegate B, Gore JC, Eward K, Signorile JF. Comparison of MRI with EMG to study muscle activity associated with dynamic plantar flexion. Magn Reson Imaging. 2003 Oct;21(8):853-61.

31. Arampatzis A, Karamanidis K, Stafilidis S, Morey-Klapsing G, DeMonte G, Brüggemann GP. Effect of different ankle- and knee-joint positions on gastrocnemius medialis fascicle length and EMG activity during isometric plantar flexion. J Biomech. 2006;39(10):1891-902.

32. Gigante A,Moschini A,Verdenelli A,Del Torto M,Ulisse S,de Palma L. Open versus percutaneous repair in the treatment of acute Achilles tendon rupture: a randomized prospective study. Knee Surg Sports Traumatol Arthrosc. 2008 Feb;16(2):204-9.

33. Chan AP, Chan YY, Fong DT, Wong PY, Lam HY, Lo CK, Yung PS, Fung KY, Chan KM. Clinical and biomechanical outcome of minimal invasive and open repair of the Achilles tendon. Sports Med Arthrosc Rehabil Ther Technol. 2011 Dec 20;3(1):32.

34. Naim F, Simşek A, Sipahioğlu S, Esen E, Cakmak G. Evaluation of the surgical results of Achilles tendon ruptures by gait analysis and isokinetic muscle strength measurements. Acta Orthop Traumatol Turc. 2005;39(1):1-6.

35. Möller M, Lind K, Movin T, Karlsson J. Calf muscle function after Achilles tendon rupture. A prospective, randomised study comparing surgical and non-surgical treatment. Scand J Med Sci Sports. 2002 Feb;12(1):9-16.

36. Jallageas R, Bordes J, Daviet JC, Mabit C, Coste C. Evaluation of surgical treatment for ruptured Achilles tendon in 31 athletes. Orthop Traumatol Surg Res. 2013 Sep;99(5):577-84.

37. Bevoni R, Angelini A, D'Apote G, Berti L, Fusaro I, Ellis S et al. Long term results of acute Achilles repair with triple-bundle technique and early rehabilitation protocol. Injury 2014 Aug;45(8):1268-74.

38. Olsson N, Nilsson-Helander K, Karlsson J, Eriksson BI, Thomée R, Faxén E, Silbernagel KG. Major functional deficits persist 2 years after acute Achilles tendon rupture. Knee Surg Sports Traumatol Arthrosc. 2011 Aug;19(8):1385-93.

39. GeremiaJM, Bobbert MF, Casa Nova M, Ott RD, LemosFde A, LupionRde O, Frasson VB, Vaz MA. The structural and mechanical properties of the Achilles tendon 2 years after surgical repair. Clin Biomech (Bristol, Avon) 2015 Jun;30(5):485-92

40. HorstmannT, Lukas C, Merk J, Brauner T, Mündermann A. Deficits 10-years after Achilles tendon repair. Int J Sports Med. 2012 Jun;33(6):474-9. 